\title{
Pregnancy and COVID-19: A Review
}

\author{
Fariha Altaf ${ }^{1}$, Amra Farrukh², Snehal Sanjay Chavan ${ }^{3}$, Yakub Kasam Sayyad ${ }^{4}$ \\ ${ }^{1}$ MBBS, MCPS, FCPS, MRCOG, MRCPI, Senior Specialist Ob Gyn, Arrayan Hospital, \\ Dr Sulaiman Al Habib Medical Group, Riyadh, Saudi Arabia \\ ${ }^{2}$ FCPS, MRCS, Specialist Paediatric Surgery, \\ ${ }^{3}$ MBBS, DGO, FOGSI-ICOG, Fellowship in Reproductive Medicine-IVF Consultant-Sprouting Seeds IVF \\ Centre \\ ${ }^{4}$ Affiliated with Shifa Clinic \\ Corresponding Author: Fariha Altaf
}

DOI: https://doi.org/10.52403/ijhsr.20220238

\begin{abstract}
The implications of COVID-19 on pregnant women are a scarcely researched topic that requires attention. The risks of the disease are a major issue for the pregnant woman and its foetus, requiring a deep and thorough understanding of the conditions that the patients go through after they are infected. This review is a comprehensive study of pregnancy and COVID-19, providing a cognisance of the pathophysiology of the disease on women during pregnancy. It has also summarised the way infection affects the body, acknowledging the changes and effects of a typical female during pregnancy. The study has primarily focused on infection from SARS-CoV-2, and highlighted the infections in contrast to MERS-CoV. The study shows that the infection triggers a cytokine storm in the human body, giving rise to several immune responses that cause changes in immune cells, including leukocytes. The complications thus arise require attention and have been addressed in this study. The lack of unequivocal evidence limits the study to a great extent, making it difficult to draw conclusions; however, notable remarks on prevention and precautionary measures have been entailed in the study. The review concludes by discussing vaccination for pregnancy as a necessary measure going forward.
\end{abstract}

Keywords: SARS-CoV-2, COVID-19, Pregnancy, Neonates, SARS, MERS, Vertical transmission, vaccination

\section{INTRODUCTION}

The advent of Coronavirus disease (COVID-19) has been detrimental for people, healthcare systems and societies globally. On $11^{\text {th }}$ March, 2020, World Health Organisation (WHO) declared COVID-19 a pandemic that was caused by Severe Acute Respiratory Syndrome Coronavirus 2 (SARS-CoV-2) (1). Most scholars have studied the effects of virus on individuals, aiming to determine several patterns in the infected people, including pregnant women. There are certain specific aspects of COVID-19 and pregnancy that obstetrics should know to diagnose the disease and understand the severity of the infection (2). In addition, they must take the necessary managerial decisions for an evidence-based protocol for managing COVID-19 in pregnancy (3). A growing concern is the implications of COVID-19 on pregnancy and associated risks of vertical transmission of the disease (4). Although pregnant women are not at an increased risk directly, however, certain diseases like heart disease, diabetes, hypertension, asthma and immunocompromised conditions like HIV and SLE greatly increase the risk of complications in pregnancy (5).

Pregnant women are comparatively at high risk for predisposing illness from COVID-19 in comparison to women who are not. There is a probability of complications like pre-term delivery (before 
37 weeks) and in some cases, even stillbirth in persons who have been infected with COVID-19 during pregnancy (6). According to a research, there is no evidence of miscarriage or pregnancy loss, and no evidence of vertical transmission of COVID-19 from mother to baby, including breast feeding (5). People with comorbidities and other factors, like age have potential for increased COVID-19 implications like Severe Acute Respiratory Syndrome (SARS) and Middle Eastern Respiratory Syndrome (MERS) while they are pregnant or post-pregnant (within 42 days of delivery). To reduce spread in pregnant women and healthcare workers, it is essential that we suspend routine checkups and replace it with video consultation or telephonic conversations wherever possible (5).

In pregnancy, there are changes in hormonal levels and function of immune system that significantly makes pregnant women more vulnerable to infections. Mortality rates and complications associated with viral infections are increased in pregnant women when compared to normal population (7). The physiological and immunological changes, like increased consumption of oxygen, alteration in $\mathrm{T}$ lymphocytic immunity, decrease in chest compliance and functional residual capacity leading to increased maternal and foetal morbidity and mortality. Moreover, pregnant women with pneumonia have high risk of delivering a low-birth weight or preterm baby (4).

To predict the implications of rising infections on the embryo or foetal is challenging, as it depends on various factors like agent, gestational timing of infection, and maternal-foetal interface (host factor). $\mathrm{Li}$ et al. (8) was the first study to evaluate COVID-19 impact on pregnancy and pneumonia using case-control study in China, and their results stated that the flu typically caused mild respiratory symptoms, of which many were asymptomatic until hospitalized. In addition, there were a few cases of premature delivery in COVID-19 pregnant women. Study also indicates that a patient who developed severe pneumonia at pregnancy of 34 weeks delivered a still baby and had drop in cardiopulmonary status, eventually undergoing multiple organ system dysfunction syndrome which required extra-corpeal membrane oxygenation (8).

It is noteworthy, despite all studies available; there is still a lack of evidence that estimates the impact of COVID-19 on pregnant women. The rapid mutations evidently witnessed by the virus are unpredictable and challenge the consistency of any impending study, questioning their value. Therefore, studies must be encouraged for considering inclusion of pregnant women in developing evidencebased research on the implications of COVID-19.

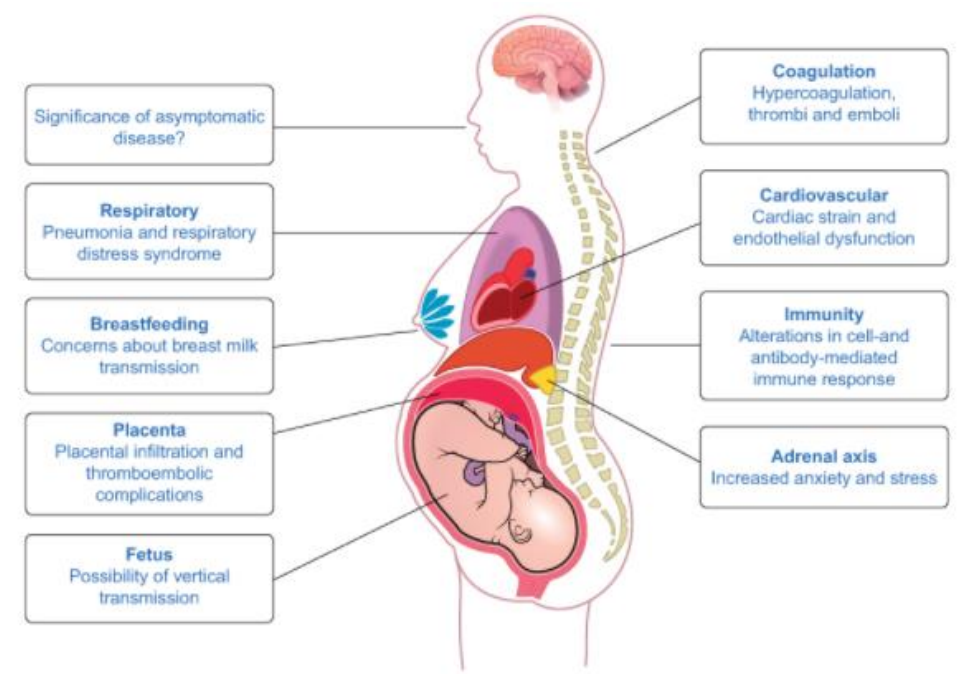

Figure 1 Effect of COVID-19 on various systems of pregnant women (5) 


\section{Pathophysiology of COVID-19 during pregnancy}

\section{a) Immune response:}

Several researches have noted the virus to be encapsulated, with evidences showing a single stranded RNA (5). Like other types of virus, immune response to coronavirus depends on the immune system of an individual; this includes the notion that the standing of COVID-19 on this range is still not clear. Toto understand the implications of coronavirus on a pregnant woman, it is essential to first get acquainted with the pathophysiology and molecular mechanisms of the virus and assess it with reference to the immune response of the mother $(5,9,10)$.

Transmission of SARS-CoV-2 virus via droplets or aerosols by respiratory route infects the pulmonary cells through SARS$\mathrm{CoV}$ receptor angiotensin-converting enzyme 2 (ACE2) which utilizes transmembrane serine protease 2 (TMPRSS2) for priming by $S$ protein $(11,12)$. Viral replication is later followed by virus release leading to pyroptosis, which means the host cell transmits stimulus that is pathological in nature, causing inflammation-mediated programmed cell death (13). Moreover, it releases DAMPs damage associated molecular patterns, ATP and nucleic acids which activates the neighbouring cells to inflammatory response leading to production of type-1 interferons, C-X-C motif chemokine 10 (CXCL10) and IL-6 (14). The SARS-CoV-2 virus causes inflammation which leads to cytokine storm resulting in failure of multiple organs (5, 13). For patients who have symptoms that are mild in nature, it is likely that the immune system responds aptly to infection. In pregnancy, the virus causes alteration in immune response causing the following:

i. There is a significant increase in Th1 and Th2 response in defence of SARSCoV-2 virus when studying the pathogenesis of severe form of COVID19 disease (5).

ii. There is a substantial decrease in natural killer cells (NK) in pregnant women which is thought to be an innate immunity's crucial factor in clearance of the virus. Also, the clinical impact of the decrease in these cells is still not evident.

iii. Pregnant women had less plasmacytoid dendritic cells (PDCs) which were vital in production of type 1 interferon (5).

iv. Progesterone levels were found to be on higher side that functioned to repair the lung damage. More research on changes pertaining to pregnancy and its effect on hormonal levels is anticipated (5).

\section{b) Respiratory system response:}

There is a change of shape of chest elevating the diaphragm which alters the function of respiratory system. Since there is a drop in total lung capacity and failure of clearing secretions, it can make pregnant women more prone to causing severe infection of the respiratory system $(5,15)$.

\section{c) Coagulation response:}

Factors of coagulation and plasmin levels are elevated in pregnancy leading them to an increased chance of developing thromboembolic diseases that result in mortality cases (16).

\section{d) Endothelial cell function:}

Acute respiratory distress syndrome (ARDS) is the primary factor causing mortality in COVID-19 patients. Furthermore, pulmonary endothelial cell dysfunction plays a vital role in onset and progression in ARDS (5).

\section{e) Placental response:}

COVID-19 infection has impact on transmission via placenta, though its studies are not yet evidently studied. Even though there are chances of vertical transmission, the mechanism that follows this type of transmission is yet not clear, entailing the need for further study on the topic (5).

\section{Effect of COVID-19 on pregnant women}

The spread of coronavirus occurs through sneezing, coughing or interaction 
with positive patients which enters via either eyes, nose or oral route passing into the respiratory system through ACE-2 receptors (17). Few case studies and follow-ups discussed in the table below demonstrate maternal and neonatal effect after COVID19 infection. Severity has been reported in each case ranging from $41 \%$ in MERS with mortality rate of $25 \%$ and $35 \%$ in SARS with mortality rate of $18 \%$ in pregnant women who eventually required mechanical ventilation (18). According to Yam et al. (17), preterm deliveries have been recorded in the range of $18-43 \%$. Maternal vascular malperfusion (MVM) has been recorded in patients including atherosis and fibroid necrosis and mural hypertrophy (17).

In order to receive appropriate and timely treatment, antenatal care is considered to be important for pregnant women, especially when the case is severe. Although frequent visits to the hospital increase chances of the patient contacting the virus, it is advisable that pregnant women resort to online consultation to significantly decrease possible contact with the virus $(17,19)$. Blood pressure must be checked at home, following several routine investigation procedures that are essential for expecting women. Because there is no appropriate study indicating the vertical transmission of SARS-CoV-2, no objection to vaginal delivery is seen until the foetus is compromised (19). Furthermore, several studies have noted that breastfeeding also takes a hit, advocating significant positives of breast feeding that include allergy protection and immunity provided by $\operatorname{IgA}$ that is present in breast milk that fights against gastrointestinal and respiratory infections. Guidelines that help new mothers include that the mothers should cover face while breast feeding and wash hands thoroughly before feeding (17).

\section{Clinical Features of COVID-19}

Signs and symptoms of SARS-CoV and MERS-CoV have similar outlook, even though ARDS is most common in MERS (4). Main clinical factors include pyrexia, myalgia, dry cough, headache, dyspnoea, vomiting, diarrhoea, nausea, pneumonia and dizziness. Fatality rate is much high in pregnant women with MERS infection where severe complications include stillbirths. Transmission of virus is mainly by aerosols, faecal and oral route. Radiological features include bilateral ground-glass or patchy opacities (4).

Diagnostic tests on COVID-19 have surfaced that lymphopenia occurs in the body; followed by increased prothrombin time (PT), increase in lactate dehydrogenase (LDH), high alanine aminotransferase (ALT) and an increase in aspartate aminotransferase (AST) are the few observations (20-22). In addition, the changes observed include increase in Ddimer, increased neutrophils, a rise in Creactive protein (CRP), and high values of troponin (23). These indications are frequently ones observed in all cases of COVID-19, including its variants. Reverse transcriptase - polymerase chain reaction (RT-PCR) is the best method for typically diagnosing COVID-19 and identifying whether an individual is infected or not (24). Various studies, like Wang et al. (20) and Huang et al. (21) have noted the manner in which RT-PCR must be conducted, followed by its frequency and repetition to get accurate results (25).

Omicron and pregnancy: In South Africa a new variant of Covid-19 was found in the late November 2021 and WHO categorized it as the variant of concern (26). The spread and epidemiology of this variant is still not evident. Studies demonstrate that this variant is more transmissible than other variant and involves significant evasion in the immune system. The healthcare officials have focused on precautionary measures for both pregnant and high-risk individuals to fight Covid-19. However, the spread of message indicating it as "mild" can be misleading. Centres for disease Control and Prevention (CDC) enlists fever, cough, difficulty in breathing, fatigue, headache and body ache, loss of taste/smell, sore throat, runny nose, nausea and vomiting as 
the symptoms. These are the symptoms found in both pregnant and other individuals. Pregnant women have suppression of immune system and contacting with the virus can have ill-effects on the baby. Irrespective of the type of variant, pregnant women are at an increased risk of getting hospitalized and even require intensive care unit (ICU) than non-pregnant individuals. Status of vaccination is utmost important in pregnant women since studies highlights that there were increase in number of pregnant women in ICU who were not vaccinated. Moreover, women who were vaccinated with booster dose had comparatively less chance of having serious impacts (26).

\section{Management and prevention}

To prevent or treat some of the maternal and foetal conditions, prescribing the accurate and safe medicines involves a vital role of obstetricians.

Obstetric and gynaecologist plan on treatment plan of pregnant women contacted by SARS-CoV virus by administration of medications. The categories of medicine include NSAIDs, MgSO4 and antenatal corticosteroid (27).

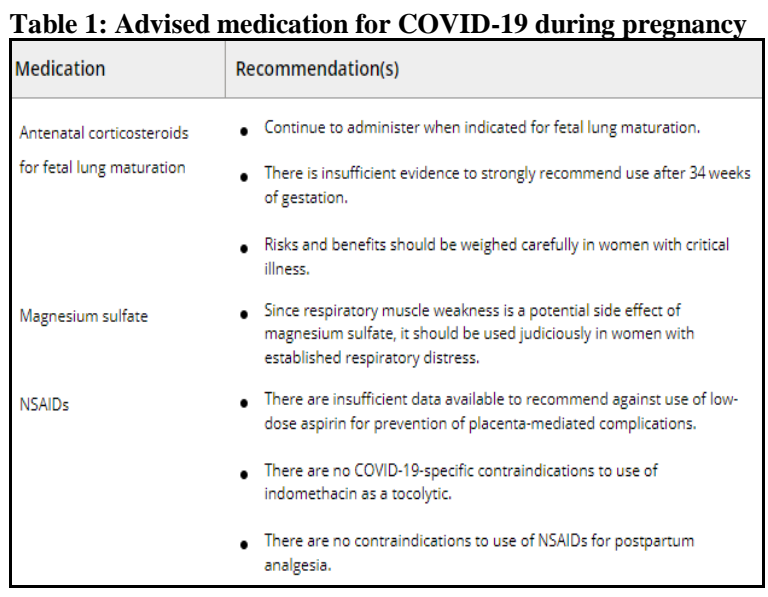

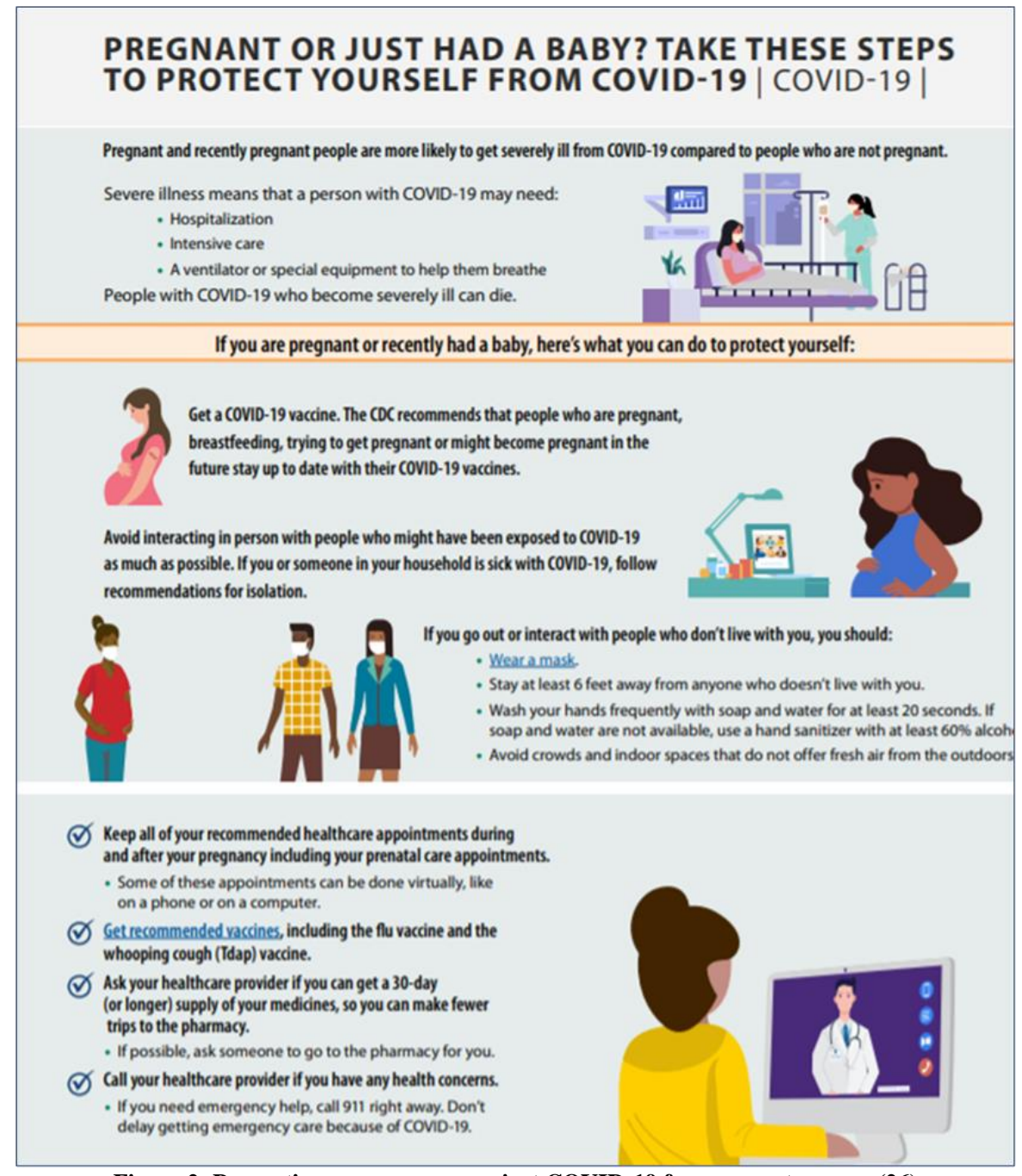

Figure 2: Precautionary measures against COVID-19 for pregnant women (26) 
Since the transmission of the virus is mainly through the spread of droplets, it is imperative that hospitals, healthcare centres, camps and clinics manage the cases and keep pregnant women at bay from COVID19 (4). Even though vertical transmission is not the case identified in pregnant women, it is still important to take utmost precautions for saving miscarriage, preterm delivery, intrauterine growth restriction and endotracheal intubation. It is imperative to avoid admission to the neonatal intensive care unit. The limited data makes it difficult to draw conclusions, however, it is noteworthy that MERS infections have a high tendency to cause unfavourable clinical results in expecting patients, including their children, during pregnancy $(4,28)$.

\section{Vaccination in pregnancy}

With the rising number of vaccination programmes throughout the world, many women are still doubtful about its effect on fertility (29). Although pregnant women were not included in the clinical trial of vaccination drive. A research done by the scientist at Pfizer stated in his article that the antibodies present in the COVID-19 vaccine might attack the placenta. The antibodies which recognized SARS-CoV-2 protein can cross react with human placental protein syncytin 1 and resulting in damaging the placenta (30). It was believed to be the blow of rumour claiming the vaccine to affect fertility and pregnancy. Although studies were carried out in early pregnant cases and control group, stating that no evident differences were found in vaccinated pregnant women like miscarriages or any other harmful effect (29).

COVID-19 vaccine is expected to mitigate the risks of the virus. Being vulnerable to infections and diseases, pregnant women will more likely need intensive care if they acquire COVID-19. In some cases, doctors may have to choose for early delivery of babies, resulting in them getting admitted to the neonatal unit (31). Therefore, like other vulnerable populations such as older people and infants, pregnant women must be prioritized for vaccination. Now the question that arises is - Is COVID19 vaccination safe during pregnancy? It is reported that, given the increased hazardous risks associated with COVID-19, many pregnant women have opted to get vaccinated (29).

Nevertheless, there needs to be more research done on what outcomes has the vaccination led to for these women and their babies. However, CDC, WHO and RCOG have recommended vaccination as mandatory for all pregnant women. More data will allow us to consider all outcomes and decide whether to increase the reach of vaccination to more pregnant women. And until then, those women planning to be pregnant can be confident that all evidence shows that vaccination has nothing to do with infertility(26).

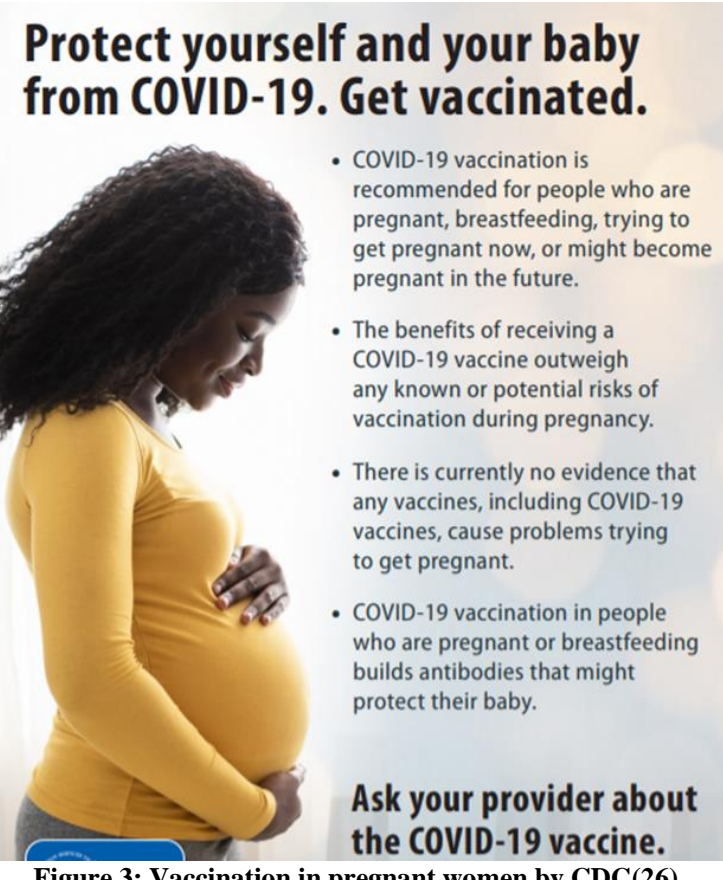

Governmental authorities of most countries and WHO promote vaccination and boosters for pregnant women due to the known risks of severe COVID-19. Therefore, vaccination is a debatable topic today, and along with the other research, it requires robust evidence to support it. Although no notable studies have remarked on negative results from the study, there is 
still a need for post-vaccine surveillance data that will prove vital to understanding abnormalities in babies. This includes longterm results indicative of problems and issues in babies going forward due to vaccination. The study has provided a comprehensive literature on the implications of COVID-19 on pregnancy, followed by an understanding of the effects of the disease on expecting women. It has studied pathophysiology of COVID-19 during pregnancy, providing a rationale of its effects on human body. Furthermore, the study has analysed the clinical features of COVID-19 from several academic sources available, to understand the key changes in the human body during infection. In addition, the study has provided an understanding on the management and prevention of coronavirus for pregnant women, including the possibilities of vaccination.

It is noteworthy that data available is sparse, requiring more research on pregnant women to draw clarity on the matter. Many regulatory bodies have recommended the use of vaccination on pregnant women, after conducting rigorous research. The possible implications are yet unknown, as the topic is still under scrutiny. This includes no notion of 'red flags', indicating a healthy status of foetus and no premature delivery. As there is no cure yet available, vaccination is the only available remedy, along with precaution. Pregnant women are required to take extensive care and precautionary measures that address any future variants to protect their babies.

Definite conclusions are still difficult to draw because of limited studies and the widespread implications of COVID19 in every way. The lack of adequate and robust information is indicative of the fact that risk factors are several and plausible justifications limited. New-born testing may possible going forward, including the likeability of vertical transmissions, however, prevention is the best means to keep COVID-19 away.

\section{CONCLUSION}

Future research on deleterious effects in babies are impending, to identify whether this effect is limited to pregnant women or spreads to their foetus. Education is key in reducing risks and promoting awareness with regards to the effects of the disease on expecting women, however, more research must be encouraged by governmental authorities of countries to understand the ways in which COVID-19 affects the human body during pregnancy. This also includes collecting and analysing post-vaccination records of pregnant women for understanding its long-term implications on babies in the future.

\section{Acknowledgement: None}

\section{Conflict of Interest: None}

\section{Source of Funding: None}

\section{REFERENCES}

1. Ciotti M, Ciccozzi M, Terrinoni A, Jiang W-C, Wang C-B, Bernardini S. The COVID-19 pandemic. Critical Reviews in Clinical Laboratory Sciences. 2020;57(6):365-88.

2. Kasaven LS, Saso S, Barcroft J, Yazbek J, Joash K, Stalder C, et al. Implications for the future of obstetrics and gynaecology following the COVID-19 pandemic: a commentary. BJOG: An International Journal of Obstetrics \& Gynaecology. 2020;127(11):1318-23.

3. Schwartz DA. The effects of pregnancy on women with COVID-19: Maternal and infant outcomes. Oxford University Press US; 2020. p. 2042-4.

4. Wenling Y, Junchao Q, Xiao Z, Ouyang S. Pregnancy and COVID-19: management and challenges. Revista do Instituto de Medicina Tropical de São Paulo. 2020;62.

5. Wastnedge EA, Reynolds RM, Van Boeckel SR, Stock SJ, Denison FC, Maybin JA, et al. Pregnancy and COVID-19. Physiological reviews. 2021;101(1):303-18.

6. services USDoHAH. Pregnant People: U.S Department of Health And Human services; 2021 [Available from: https://www.cdc.gov/coronavirus/2019- 
ncov/need-extra-precautions/pregnantpeople.html.

7. Takemoto ML, Menezes MO, Andreucci CB, Knobel R, Sousa LA, Katz L, et al. Maternal mortality and COVID-19. The Journal of Maternal-Fetal \& Neonatal Medicine. 2020:1-7.

8. Alberca RW, Pereira NZ, Oliveira LMDS, Gozzi-Silva SC, Sato MN. Pregnancy, viral infection, and COVID-19. Frontiers in immunology. 2020;11:1672.

9. Jamieson DJ, Theiler RN, Rasmussen SA. Emerging infections and pregnancy. Emerging infectious diseases. 2006;12(11): 1638 .

10. Schjenken JE, Tolosa JM, Paul JW, Clifton VL, Smith R. Mechanisms of maternal immune tolerance during pregnancy. Recent advances in research on the human placenta. 2012;11:211-42.

11. Cheng VC, Wong S-C, Chen JH, Yip CC, Chuang VW, Tsang OT, et al. Escalating infection control response to the rapidly evolving epidemiology of the coronavirus disease 2019 (COVID-19) due to SARSCoV-2 in Hong Kong. Infection Control \& Hospital Epidemiology. 2020;41(5):493-8.

12. Jiang G, Wang C, Song L, Wang X, Zhou $\mathrm{Y}$, Fei $\mathrm{C}$, et al. Aerosol transmission, an indispensable route of COVID-19 spread: case study of a department-store cluster. Frontiers of Environmental Science \& Engineering. 2021;15(3):1-12.

13. Jose RJ, Manuel A. COVID-19 cytokine storm: the interplay between inflammation and coagulation. The Lancet Respiratory Medicine. 2020;8(6):e46-e7.

14. Nile SH, Nile A, Qiu J, Li L, Jia X, Kai G. COVID-19: Pathogenesis, cytokine storm and therapeutic potential of interferons. Cytokine \& growth factor reviews. 2020;53:66-70.

15. Goodnight WH, Soper DE. Pneumonia in pregnancy. Critical care medicine. 2005; 33(10):S390-S7.

16. Ji H-L, Zhao R, Matalon S, Matthay MA. Elevated plasmin (ogen) as a common risk factor for COVID-19 susceptibility. Physiological reviews. 2020.

17. Yam C, Jokubaitis V, Hellwig K, Dobson R. MS, pregnancy and COVID-19. Multiple Sclerosis Journal. 2020;26(10):1137-46.

18. Dashraath P, Wong JLJ, Lim MXK, Lim LM, Li S, Biswas A, et al. Coronavirus disease 2019 (COVID-19) pandemic and pregnancy. American journal of obstetrics and gynecology. 2020;222(6):521-31.

19. Campbell KH, Tornatore JM, Lawrence KE, Illuzzi JL, Sussman LS, Lipkind HS, et al. Prevalence of SARS-CoV-2 among patients admitted for childbirth in Southern Connecticut. Jama. 2020;323(24):2520-2.

20. Wang D, Hu B, Hu C, Zhu F, Liu X, Zhang $\mathrm{J}$, et al. Clinical characteristics of 138 hospitalized patients with 2019 novel coronavirus-infected pneumonia in Wuhan, China. Jama. 2020;323(11):1061-9.

21. Huang C, Wang Y, Li X, Ren L, Zhao J, Hu $\mathrm{Y}$, et al. Clinical features of patients infected with 2019 novel coronavirus in Wuhan, China. The lancet. 2020;395(10223):497-506.

22. Siordia Jr JA. Epidemiology and clinical features of COVID-19: A review of current literature. Journal of Clinical Virology. 2020;127:104357.

23. Guan W-j, Ni Z-y, Hu Y, Liang W-h, Ou C$\mathrm{q}$, He J-X, et al. Clinical characteristics of 2019 novel coronavirus infection in China. MedRxiv. 2020.

24. Fang Y, Zhang H, Xie J, Lin M, Ying L, Pang P, et al. Sensitivity of chest CT for COVID-19: comparison to RT-PCR. Radiology. 2020;296(2):E115-E7.

25. Ai T, Yang Z, Hou H, Zhan C, Chen C, Lv $\mathrm{W}$, et al. Correlation of chest CT and RTPCR testing for coronavirus disease 2019 (COVID-19) in China: a report of 1014 cases. Radiology. 2020;296(2):E32-E40.

26. Miller K. How Does the Omicron Variant Affect Pregnant Women? USA: What to Expect.; 2022 [Available from: https://www.whattoexpect.com/pregnancy/p regnancy-health/omicron-variant-pregnantwomen.

27. D'Souza R, Ashraf R, Rowe H, Zipursky J, Clarfield L, Maxwell C, et al. Pregnancy and COVID-19: pharmacologic considerations. Ultrasound in Obstetrics \& Gynecology. 2021;57(2):195-203.

28. Park MH, Kim HR, Choi DH, Sung JH, Kim JH. Emergency cesarean section in an epidemic of the middle east respiratory syndrome: a case report. Korean journal of anesthesiology. 2016;69(3):287.

29. Male V. Are COVID-19 vaccines safe in pregnancy? Nature Reviews Immunology. 2021;21(4):200-1.

30. Cosma S, Carosso AR, Cusato J, Borella F, Carosso M, Bovetti M, et al. Coronavirus 
disease 2019 and first-trimester spontaneous abortion: a case-control study of 225 pregnant patients. American journal of obstetrics and gynecology. 2021;224(4): 391. e1-. e7.

31. Allotey J, Stallings E, Bonet M, Yap M, Chatterjee S, Kew $\mathrm{T}$, et al. Clinical manifestations, risk factors, and maternal and perinatal outcomes of coronavirus disease 2019 in pregnancy: living systematic review and meta-analysis. Bmj. 2020;370.

How to cite this article: Fariha Altaf, Amra Farrukh, Chavan SS et.al. Pregnancy and COVID-19: a review. Int J Health Sci Res. 2022; 12(2): 271-279. DOI: https://doi.org/10. 52403/ijhsr.20220238 